\title{
Quantitative MRI Reveals Grey Matter Abnormalities in Children With Drug-Naïve Attention- Deficit/Hyperactivity Disorder
}

\section{Shu Su}

Sun Yat-sen University First Affiliated Hospital

\section{Yingqian Chen}

Sun Yat-sen University First Affiliated Hospital

\section{Yan Dai}

Sun Yat-sen University First Affiliated Hospital

\section{Liping Lin}

Sun Yat-sen University First Affiliated Hospital

\section{Long Qian}

GE Healthcare Beijing

Qin Zhou

Sun Yat-sen University First Affiliated Hospital

\section{Mengsha Zou}

Sun Yat-sen University First Affiliated Hospital

Hongyu Zhang

Sun Yat-sen University First Affiliated Hospital

Meina Liu

Sun Yat-sen University First Affiliated Hospital

\section{Xianhong Xiang}

Sun Yat-sen University First Affiliated Hospital

Zhiyun Yang ( $\nabla$ yzhyun@mail.sysu.edu.cn )

Sun Yat-sen University First Affiliated Hospital https://orcid.org/0000-0002-6139-9049

\section{Research Article}

Keywords: attention-deficit/hyperactivity disorder, quantitative MRI, grey matter

Posted Date: April 23rd, 2021

DOI: https://doi.org/10.21203/rs.3.rs-349668/v1 
License: (c) (i) This work is licensed under a Creative Commons Attribution 4.0 International License. Read Full License 


\section{Abstract \\ Purpose}

To investigate the quantitative profiles of brain GM in pediatric drug-naïve ADHD patients using Synthetic magnetic resonance imaging (SyMRI).

\section{Method and Materials:}

A total of 37 drug-naïve pediatric ADHD and 27 age- and gender-matched healthy controls $(\mathrm{HC})$ were enrolled in this study. Each subject underwent both SyMRI and conventional 3D T1-FSPGR scans. Quantitative parameters, T1 and T2 maps, were extracted from the SyMRI data. Between-group quantitative maps were compared using a general linear model analysis. Pearson correlation analysis was conducted to assess the association between significantly altered MR indices and clinical measurements in ADHD.

\section{Results}

Compared with the $\mathrm{HC}$ group, altered $\mathrm{T} 1$ and $\mathrm{T} 2$ relaxometry times in the ADHD group were mainly distributed in GM regions of the cerebellum, attention and execution control network, default mode network, and limbic areas. Moreover, the T1 value of the right cerebellum 8 was negatively correlated with the attention concentration level in ADHD $(\mathrm{R}=0.1401, P=0.0225)$. With regards to T2 map, the associations were observed between the attention level of ADHD patients and left fusiform gyrus $(R=$ $0.2509, P=0.0016)$, and right cerebellum crus2 $(\mathrm{R}=0.1422, P=0.0214)$.

\section{Conclusion}

Altered T1, T2 values found in specific regions of GM may reveal widespread micromorphology changes, i.e., brain iron deficiency, low myelin content, and enlarged vascular interstitial space in ADHD patients. Thus, T1, T2 values might be promising imaging markers for future ADHD studies.

\section{Introduction}

Attention-deficit/hyperactivity disorder (ADHD) is a neurodevelopmental disorder that commonly occurs in children and adolescents. ADHD is usually characterized by age-inappropriate inattention, hyperactivity, and impulsivity $[1,2]$. So far, several neuropsychological, genetic testing, and neurochemical studies have attempted to explain the pathophysiology of ADHD [3]. Nevertheless, the neural dysfunction leading to ADHD still remains elusive. Increasing neuroimaging findings have suggested that ADHD is associated with abnormal grey matter (GM) anatomy and aberrant neural circuit activity. Structural magnetic resonance imaging (MRI) indicated structural alterations of cortical and subcortical regions in ADHD 
patients, including decreased cortical and subcortical volume, reduced cortical thickness, reduced cortical surface area, and gyrification [4-6]. According to task- or resting- functional MRI studies, aberrant neural circuit activity is mainly located in line with the frontostriatal pathway dysfunction and default mode network dysfunction [6-8]. Though structure, MRI allows macromorphological measurements of the brain. The functional MRI can reveal neural cortical activity. Yet, the microscopic biophysical processes underlying the macroscopic changes in ADHD remain unclear.

Recent studies indicated that the characterization of these processes could be realized using quantitative MRI biomarkers of brain tissue microstructure $[9,10]$. Quantitative MR techniques have been used to study multiple central nervous system conditions by providing useful information for assessing diseases' microscopic biophysical processes [11-13]. However, so far, no studies have explored T1, T2 relaxation times of the brain pathophysiology in ADHD, where brain microstructure abnormalities could potentially be uncovered.

It has been hypothesized that brain differences observed in ADHD are due to a delay in maturational processes. Myelin has a key role in brain microarchitectural changes. Quantitative MRI parameters provide well-defined physical parameters and can be applied to estimate the human brain's development $[13,14]$. Specifically, the T1 relaxation time is affected by myelin, water, and iron concentrations in brain tissue; myelin content affects the T1 value, and iron deficiency prolongs the T1 value [14]. T2 is a common water proton relaxometry measure, which can estimate the myelin water fraction (MWF), defined as the ratio between the short T2 components of myelin water (10-40 milliseconds) and the total area of the T2 distribution [15]. Due to the brain's different physical properties and microstructural environments, water between myelin layers has a shorter T2 time than nonmyelin water [15]. In general, T2 relaxometry is mainly influenced by tissue myelin content, while T1 relaxation times are related mainly (but not only) to the microstructural tissue integrity, including myelin content and iron content. In the other neurodevelopmental disorders on the autism spectrum, increased T2 of the brain was found to reflect reduced myelin content and correspondingly increased water content [11].

Many different relaxometry techniques have been applied to evaluate brain microstructure in children. In most cases, T1, T2 values were obtained using separate methods, which resulted in excessive acquisition time. Each sequence of T1 mapping or T2 mapping usually takes minutes, while SyMRI offers a B1corrected T1 map and a T2 map in 5-6 min [16], which makes it possible to estimate the T1 and T2 values during a single scanning session. Previous studies have demonstrated high repeatability and reproducibility of the SyMRI parametric maps across multiple sites and time points [16] and a high comparability of their constructed contrast-weighted images to conventional MR images in the brain examination of children [17] and normal subjects, as well in the assessment of different neurologic conditions $[17,18]$. In this study, the synthetic MRI was applied to investigate brain microstructure alterations of grey matter associated with ADHD using a whole-brain approach. In addition, we also correlated these significantly altered MR parameters with the disease symptom severity (Test of Variables of Attention) to evaluate if these changes could reflect the disease level. Based on the above literature, we assumed that the ADHD patients would display cortical GM microstructure alterations. Furthermore, we 
intended to investigate whether the significantly altered brain regions were correlated with clinical measurements.

\section{Materials And Method}

\section{Participants}

A total of 37 pediatric ADHD patients and 27 healthy pediatric healthy volunteers were enrolled in this study. The 37 ADHD patients included 14 cases of the inattention subtype, 23 cases of the combined subtype, but no case of the hyperactivity-impulsivity subtype. The age range of the enrolled participants was 6-13y, with an average age of 9y. Age, gender, and handedness were comparable between the two groups. The male to female ratio in both groups was 6:1 (males: females in the ADHD group and healthy control group). All of the ADHD patients were drug-naïve.

This study was approved by the institutional review board of the First Affiliated Hospital of Sun Yat-sen University (No. [2019]328). Written informed consent was obtained from the guardians of all the subjects (patients) in this study.

\section{Clinical Assessment}

The patients were diagnosed with ADHD according to the criteria of the Diagnostic and Statistical Manual of Mental Disorders, fifth edition (DSM-V). Both the parent and teacher ADHD indices $\geq 75$ th percentile were considered as ADHD positive.

The typical healthy volunteers were excluded from the diagnosis of ADHD based on the same reports, with both parent and teacher ADHD indices < 75 th percentile. A face-to-face interview was conducted with all the participants to confirm diagnostic status.

\section{Image Acquisition and processing}

MRI examinations of all participants were performed on a 3.0T scanner (SIGNA Pioneer GE Healthcare, WI, USA) using 32-channel head coils. Following localizers, a coronal T2-weighted sequence was collected to rule out any cranial organic lesion. For each subject, the sagittal three-dimensional T1weighted fast spoiled gradient echo-based sequence (T1-FSPGR) with $1.00 \mathrm{~mm}$ isotropic resolution and SyMRI scan were performed. With regard to SyMRI, a two-dimensional multiple-dynamic multiple-echo (MDME) pulse sequence, comprising four automatically calculated saturation delay times and two echo times, was applied to acquire the axial sections. The parameters were as follows: $\mathrm{TR}=10205.0 \mathrm{~ms}, \mathrm{TE}=$ $11.3 \mathrm{~ms}$, flip angle $=20^{\circ}$, thickness $=2 \mathrm{~mm} /$ no gap, $\mathrm{NEX}=1.00, \mathrm{ETL}=16$, pixel size $=2.0 \mathrm{~mm} \times 2.0 \mathrm{~mm}$, scanning time $=5.5 \mathrm{~min}$.

After MR scanning, the quantitative maps $(T 1, T 2)$ were estimated from the SyMRI data using the vendorprovided postprocessing software (SyntheticMR, v11.2.2). To obtain the brain regional level T1, T2 values, the T1-FSPGR image was first co-registered to the T1 map for each subject. Then, the co- 
registered T1 images were normalized to Montreal Neurological Institute (MNI) space using FSL (www.fmrib.ox.ac.uk/fsl, v6.0). Thereafter, all the T1, T2 images could be transformed to MNI space. Finally, the AAL atlas was applied to all the normalized images to extract the values of all 116 regions, including cerebrum and cerebellum.

\section{Statistical analysis}

SPSS v21.0 (IBM Corp., Armonk, New York) was used to perform statistical analysis. The normality of the data was assessed via the Shapiro-Wilk test. We compared the demographic data using a chi-square test for all categorical variables. Between-group differences (ADHD vs. HC) of regional quantitative values were performed using an analysis of covariance (ANCOVA) with age and gender as nuisance covariates.

Pearson correlation analysis was performed between the significantly altered regional quantitative indices and clinical symptom severity measures within the ADHD group. For all tests, a $P$ value $<0.05$ was considered to be statistically significant. Importantly, a false-discovery rate (FDR) was performed to control the error of multiple comparisons.

\section{Results}

\section{Demographic Data}

There were no significant differences in age $(P=0.390)$ or gender $(P=0.772)$ between the two groups (Table 1). Cortical and subcortical regions of interest are shown in Table 2.

Table 1

Demographic data of ADHD patients and healthy control $(\mathrm{HC})$ subjects

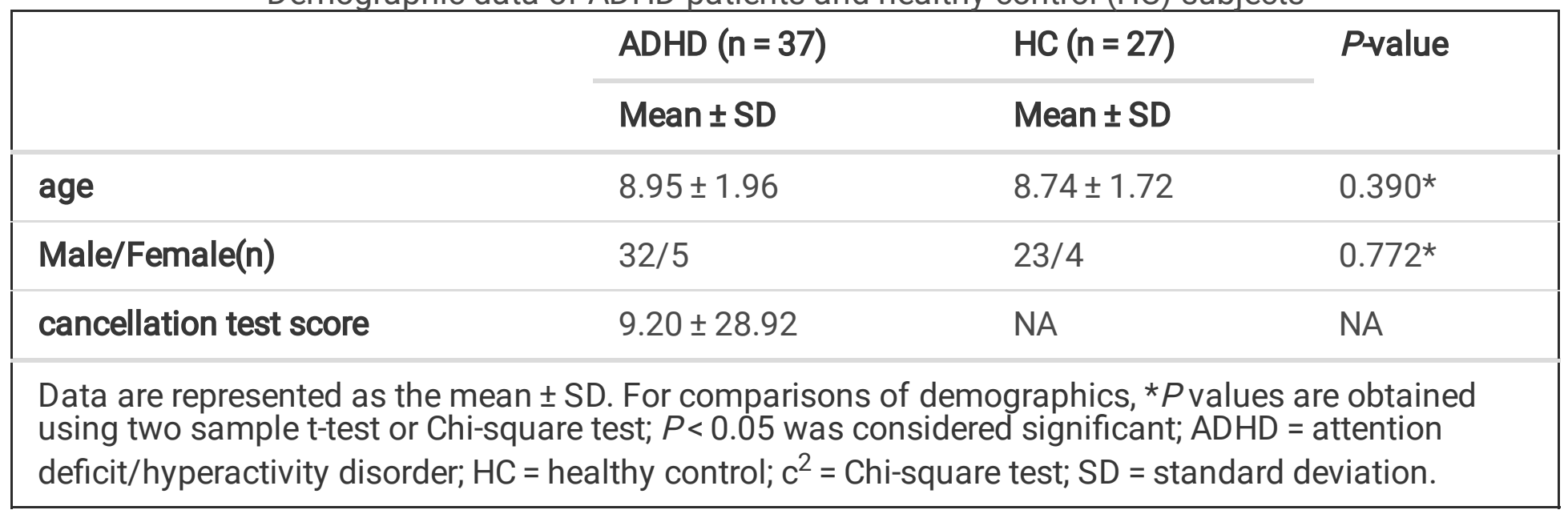


Table 2

Cortical and subcortical regions of interest defined in the current study

\begin{tabular}{|c|c|c|c|c|c|}
\hline Index & Region & Abbr. & Index & Region & Abbr. \\
\hline$(1,2)$ & Precental gyrus & PreCG & $(59,60)$ & Superior parietal gyrus & SPG \\
\hline$(3,4)$ & $\begin{array}{l}\text { Superior frontal gyrus, } \\
\text { dorsolateral }\end{array}$ & SFGdor & $(61,62)$ & $\begin{array}{l}\text { Inferior parietal, but } \\
\text { supramarginal and } \\
\text { angular gyri }\end{array}$ & IPL \\
\hline$(5,6)$ & $\begin{array}{l}\text { Superior frontal gyrus, } \\
\text { orbital part }\end{array}$ & ORBsup & $(63,64)$ & Supramarginal gyrus & SMG \\
\hline$(7,8)$ & Middle frontal gyrus & MFG & $(65,66)$ & Angular gyrus & ANG \\
\hline$(9,10)$ & $\begin{array}{l}\text { Middle frontal gyrus, } \\
\text { orbital part }\end{array}$ & ORBmid & $(67,68)$ & Precuneus & PCUN \\
\hline$(11,12)$ & $\begin{array}{l}\text { Inferior frontal gyrus, } \\
\text { opercular part }\end{array}$ & IFGoperc & $(69,70)$ & Paracentral lobule & PCL \\
\hline$(13,14)$ & $\begin{array}{l}\text { Inferior frontal gyrus, } \\
\text { triangular part }\end{array}$ & IFGtriang & $(71,72)$ & Caudate nucleus & CAU \\
\hline$(15,16)$ & $\begin{array}{l}\text { Inferior frontal gyrus, } \\
\text { orbital part }\end{array}$ & ORBinf & $(73,74)$ & $\begin{array}{l}\text { Lenticular nucleus, } \\
\text { putamen }\end{array}$ & PUT \\
\hline$(17,18)$ & Rolandic operculum & ROL & $(75,76)$ & $\begin{array}{l}\text { Lenticular nucleus, } \\
\text { pallidum }\end{array}$ & PAL \\
\hline$(19,20)$ & $\begin{array}{l}\text { Supplementary motor } \\
\text { area }\end{array}$ & SMA & $(77,78)$ & Thalamus & THA \\
\hline$(21,22)$ & Olfactory cortex & OLF & $(79,80)$ & Heschl gyrus & HES \\
\hline$(23,24)$ & $\begin{array}{l}\text { Superior frontal gyrus, } \\
\text { medial }\end{array}$ & SFGmed & $(81,82)$ & $\begin{array}{l}\text { Superior temporal } \\
\text { gyrus }\end{array}$ & STG \\
\hline$(25,26)$ & $\begin{array}{l}\text { Superior frontal gyrus, } \\
\text { medial orbital }\end{array}$ & ORBsupmed & $(83,84)$ & $\begin{array}{l}\text { Temporal pole: } \\
\text { superior temporal } \\
\text { gyrus }\end{array}$ & TPOsup \\
\hline$(27,28)$ & Gyrus rectus & REC & $(85,86)$ & Middle temporal gyrus & MTG \\
\hline$(29,30)$ & Insula & INS & $(87,88)$ & $\begin{array}{l}\text { Temporal pole: middle } \\
\text { temporal gyrus }\end{array}$ & TPOmid \\
\hline$(31,32)$ & $\begin{array}{l}\text { Anterior cingulate and } \\
\text { paracingulate gyri }\end{array}$ & ACG & $(89,90)$ & Inferior temporal gyrus & ITG \\
\hline$(33,34)$ & $\begin{array}{l}\text { Median cingulate and } \\
\text { paracingulate gyri }\end{array}$ & DCG & $(91,92)$ & Cerebellum Crus1 & CbeCru1 \\
\hline
\end{tabular}

Note: The regions are listed according to a prior template obtained from an AAL atlas; the odd number represents the corresponding brain regions in the left hemisphere; the even number denotes the specific brain regions in the right hemisphere. 


\begin{tabular}{|c|c|c|c|c|c|}
\hline Index & Region & Abbr. & Index & Region & Abbr. \\
\hline$(35,36)$ & $\begin{array}{l}\text { Posterior cingulate } \\
\text { gyrus }\end{array}$ & PCG & $(93,94)$ & Cerebellum Crus2 & CbeCru2 \\
\hline$(37,38)$ & Hippocampus & HIP & $(95,96)$ & Cerebellum 3 & Cbe3 \\
\hline$(39,40)$ & $\begin{array}{l}\text { Parahippocampal } \\
\text { gyrus }\end{array}$ & PHG & $(97,98)$ & Cerebellum 4-5 & Cbe4-5 \\
\hline$(41,42)$ & Amygdala & AMYG & $(99,100)$ & Cerebellum 6 & Cbe6 \\
\hline$(43,44)$ & $\begin{array}{l}\text { Calcarine fissure and } \\
\text { surrounding cortex }\end{array}$ & CAL & $(101,102)$ & Cerebellum 7b & Cbe7b \\
\hline$(45,46)$ & Cuneus & CUN & $(103,104)$ & Cerebellum 8 & Cbe8 \\
\hline$(47,48)$ & Lingual gyrus & LING & $(105,106)$ & Cerebellum 9 & Cbe9 \\
\hline$(49,50)$ & Superior occipital gyrus & SOG & $(107,108)$ & Cerebellum 10 & Cbe10 \\
\hline$(51,52)$ & Middle occipital gyrus & MOG & $(109,110)$ & (Vermis $1-2$, Vermis 3 ) & $\begin{array}{l}\text { Ver1-2, } \\
\text { Ver3 }\end{array}$ \\
\hline$(53,54)$ & Inferior occipital gyrus & IOG & $(111,112)$ & (Vermis 4-5, Vermis 6) & $\begin{array}{l}\text { Ver4-5, } \\
\text { Ver6 }\end{array}$ \\
\hline$(55,56)$ & Fusiform gyrus & FFG & $(113,114)$ & (Vermis 7, Vermis 8) & $\begin{array}{l}\text { Ver7, } \\
\text { Ver8 }\end{array}$ \\
\hline$(57,58)$ & Postcentral gyrus & PoCG & $(115,116)$ & (Vermis 9, Vermis 10) & $\begin{array}{l}\text { Ver9, } \\
\text { Ver10 }\end{array}$ \\
\hline $\begin{array}{l}\text { Note: T } \\
\text { represe } \\
\text { specifi }\end{array}$ & $\begin{array}{l}\text { gions are listed accor } \\
\text { he corresponding brai } \\
\text { in regions in the right }\end{array}$ & $\begin{array}{l}\text { o a } \\
\text { ons } \\
\text { pher }\end{array}$ & $\begin{array}{l}\text { te obtai } \\
\text { emisphe }\end{array}$ & $\begin{array}{l}\text { rom an } \mathrm{AAL} \text { atlas; the } \\
\text { e even number denot }\end{array}$ & $\begin{array}{l}\text { d number } \\
\text { he }\end{array}$ \\
\hline
\end{tabular}

\section{Statistical Analysis And Correlation With Clinical Variables}

There were significant differences between $\mathrm{GM}$ brain regions in $\mathrm{T} 1, \mathrm{~T} 2$ relaxation times between the ADHD and control groups. Most brain GM regions showed the longer T1 and T2 relaxation times of all GM brain microstructures in ADHD groups.

As shown in Fig. 1A and Table 3, when analyzing T1 relaxation times of individual brain GM regions, left cerebellum $7 \mathrm{~b} / 8$ and right cerebellum $7 \mathrm{~b} / 8$ had longer relaxation times in ADHD than in healthy controls. T1 relaxation times of the left superior frontal gyrus, dorsolateral part was shorter in ADHD than in healthy controls. Moreover, T1 values of the left cerebellum 8 were found to have negative correlation with attention concentration level in ADHD patients $(\mathrm{R}=0.1401, P=0.0225$, Fig. $2 \mathrm{~A})$. 
Table 3

Between-group differences of T1, T2 values between ADHD and healthy controls

\begin{tabular}{|c|c|c|c|c|c|}
\hline $\begin{array}{l}\text { ALL } \\
\text { number }\end{array}$ & Regions & $\begin{array}{l}\text { Anatomical } \\
\text { classification }\end{array}$ & Metric & Network & $\begin{array}{l}P \text {-value } \\
\text { FDR-corrected }\end{array}$ \\
\hline 3 & SFGdor.L & Prefontal & $\mathrm{T} 1$ & DMN & $<0.0001$ \\
\hline 23 & SFGmed.L & Limbic & $\mathrm{T} 2$ & DMN & 0.0232 \\
\hline 25 & ORBsupmed.L & Prefontal & $\mathrm{T} 2$ & DMN & $<0.0001$ \\
\hline 26 & ORBsupmed.R & Prefontal & $\mathrm{T} 2$ & DMN & 0.0475 \\
\hline 27 & REC.L & Paralimbic & $\mathrm{T} 2$ & DMN & 0.0341 \\
\hline 31 & ACG.L & Paralimbic & $\mathrm{T} 2$ & DMN & $<0.0001$ \\
\hline 67 & PCUN.L & Parietal & $\mathrm{T} 2$ & DMN & $<0.0001$ \\
\hline 45 & CUN.R & Occipital & $\mathrm{T} 2$ & VN & $<0.0001$ \\
\hline 46 & CUN.L & Occipital & $\mathrm{T} 2$ & VN & 0.0249 \\
\hline 55 & FFG.L & Temporal & $\mathrm{T} 2$ & VN & 0.0232 \\
\hline 8 & MFG.R & Prefontal & $\mathrm{T} 2$ & EAN & 0.0341 \\
\hline 62 & IPL.R & Parietal & $\mathrm{T} 2$ & EAN & 0.0387 \\
\hline 66 & ANG.R & Parietal & $\mathrm{T} 2$ & EAN & 0.0422 \\
\hline 19 & SMA.L & Frontal & $\mathrm{T} 2$ & SMN & 0.0145 \\
\hline 60 & SPG.R & Parietal & $\mathrm{T} 2$ & SMN & 0.0406 \\
\hline 81 & STG.L & Temporal & $\mathrm{T} 2$ & SMN & 0.0249 \\
\hline 33 & DCG.L & Paralimbic & $\mathrm{T} 2$ & Subcortical & 0.0145 \\
\hline 74 & PUT.R & Sub-cortical & $\mathrm{T} 2$ & Suncortical & 0.0145 \\
\hline 94 & rCbe Cru2 & celebellum & $\mathrm{T} 2$ & celebellum & 0.0249 \\
\hline 101 & ICbe7b & celebellum & T1 & celebellum & $<0.0001$ \\
\hline 102 & rCbe7b & celebellum & $\mathrm{T} 1 / \mathrm{T} 2$ & celebellum & $\begin{array}{l}< \\
0.0001 / 0.0145\end{array}$ \\
\hline 103 & ICbe8 & celebellum & $\mathrm{T} 1$ & celebellum & 0.0290 \\
\hline 104 & rCbe8 & celebellum & $\mathrm{T} 1$ & celebellum & $<0.0001$ \\
\hline 110 & vermis 3 & celebellum & $\mathrm{T} 2$ & celebellum & 0.0249 \\
\hline
\end{tabular}

Abbreviations: VN, visual network; EAN, attention and execution control; SMN, sensorimotor network; DMN, default mode network. P-value after FDR-corrected. The abbreviations of the regions refer to Table 
With regards to T2 maps, GM regions with higher T2 relaxation times were primarily distributed in multiple regions, such as frontal, cingulate, temporal, parietal, occipital, cuneus, and cerebellum of ADHD than healthy controls, as in the following cortical and sub-cortical regions: right middle frontal gyrus, left the supplementary motor area, left superior frontal gyrus, medial, and left superior frontal gyrus, medial orbital, etc. (Fig. 1B and Table 3). Furthermore, we observed correlation relationships between T2 relaxation times of left fusiform gyrus $(\mathrm{R}=0.2509, P=0.0016$, Fig. $2 \mathrm{~B})$ and right cerebellum crus2 $(\mathrm{R}=$ $0.1422, P=0.0214$, Fig. $2 C$ ) with attention level in ADHD patients.

\section{Discussion}

In the current study, the MR quantitative indices of GM regions were compared to show substantial differences between individuals with drug-naïve ADHD and typical healthy controls. The main differences between ADHD patients and control individuals were: 1) widespread microstructure changes in cortical and subcortical regions were observed in ADHD patients; 2) GM regions, including cerebellum, default mode network (DMN), attention and execution control network (EAN), limbic areas, etc., have been implicated in the disorder; 3) focal GM regions signal changes correlated to clinical attention concentration level were also found in our study, like left cerebellum 8, left fusiform gyrus, and right cerebellum crus2.

\section{T1, T2 values charting cortical microstructure}

As a kind of developmental disorder, ADHD has been reported to correlate with brain structure development retardation and abnormal neural network interplay $[6,7,19]$. Grey matter volume reductions in the ventrolateral prefrontal/insular-striatal regions, such as the right insula, putamen, globus pallidus, and caudate nucleus, have been found in ADHD patients [20, 21]. Voxel-based morphometry (VBM) analyses of unaffected siblings of ADHD cases have identified smaller GM volume in the prefrontal cortex, medial and orbitofrontal cortex, fronto-occipital regions, and cingulate regions in ADHD patients compared to healthy controls [22]. In our study, changes in MRI relaxometry times of these regions have also been observed in ADHD patients, which may reflect macrostructure changes and microstructure alteration in these regions.

The development of the neuron fiber myelination process has a critical role in brain development. Although myelin is more abundant in white matter, a considerable quantity of myelinated fibers can be found in cortical GM $[9,14]$. In our observation, widespread microstructure changes in cortical and subcortical regions were detected via T1 and T2 map, especially the T2 map. Increased T1value of left cerebellum $7 \mathrm{~b} / 8$ and right cerebellum $7 \mathrm{~b} / 8$ in ADHD patients can be explained by low myelin content and iron deficiency. Moreover, we also found a weak, significant negative correlation between significantly altered T1 relaxation times in the left cerebellum 8 and attention concentration level, which provide support for our assumption. For further study, more specific iron-target sequence, for example, R2*, 
susceptibility weighted imaging (SWI) or post-processing quantitative susceptibility mapping (QSM) may use to explore the between-group iron content differences.

The longer T2 relaxation times of GM found in ADHD could also be explained by low concentration of myelin; water trapped between the myelin layers has a shorter T2 relaxation time than water in the intraand extra-cellular compartments $[12,15]$. Thus, the increased T2 relaxation times of the cerebellum, EAN, DMN, and limbic areas found in our study may suggest a relatively low concentration of myelin content and increased water content. Furthermore, the significant correlation relationships between T2 relaxation times of the left fusiform gyrus and right cerebellum crus2 and the symptom severity indicated that these regions might have critical roles in ADHD pathogenesis.

Apart from GM's lower myelination degree and correspondingly increased water content, the increased vascular interstitial space due to developmental retardation may lead to signal change. In previous study, perivascular spaces had been well realized in the neurodevelopmental degeneration and neurological diseases[23], but regards to the developmental disorder, we have poor knowledge of its role related to the pathology and physiology in ADHD. Thus, further studies are needed to clarify the related pathology occurred in ADHD. Moreover, the interference caused by individual age and potential brain development bias is hard to be diminished. In summary, the quantitative MR imaging could reflect the brain tissue microstructure changes, thus furthering our knowledge of the brain changes in ADHD.

\section{The varied brain regions and the network involved}

The altered brain GM regions have been mainly classified as located in some specific functional regions $[24,25]$, including cerebellum, default mode network (DMN), attention and execution control network (EAN), limbic areas, etc. In our study, higher T2 values of right cerebellum crus II/7b, left fusiform, and higher $\mathrm{T} 1$ values of left cerebellum $7 \mathrm{~b} / 8$, right cerebellum $7 \mathrm{~b} / 8$ have been found in ADHD compared to healthy controls, which greatly overlapped with recent findings suggesting that significant gray matter network reductions occurred in bilateral crus I, left lingual gyrus, left crus II, and left fusiform in ADHD [7]. Longer T2 belongs to DMN and EAN regions, including prefrontal, parietal, cingulate, precuneus, and rectus lobes, which were also found in our study. A previous study suggested that the inverse correlation of DMN and the cognitive control networks were diminished or absent in ADHD-related children and adults $[26,27]$. In addition, a more diffuse pattern of resting-state network connectivity and delayed functional network development in children with ADHD have been reported [19].Significant increase in T2 values of limbic areas, including cingulate, insula, putamen, and temporal lobe, was also observed in ADHD, which was consistent with previous studies (meta- and mega-analysis of subcortical structural imaging studies) that found additional volume reductions in basal ganglia, insula [19,28], amygdala and hippocampus [5], as well as entire cingulate cortex structure/function abnormalities in patients with ADHD [29]. More than structure/function abnormalities, our findings indicated microstructure alterations in these regions. In general, the microstructure alteration located in the specific GM regions may be the pathophysiology basis for the structure/function abnormalities. 
In the present study, we propose that apart from GM structure/function abnormalities in ADHD, GM microstructure abnormalities may also have an important role in the underlying mechanism of ADHD. Besides, some other networks were found to be involved in having signal values alternation in our study, including the visual network (VN), sensorimotor network (SMN), and subcortical areas. However, these other regions were rather limited and thus are not discussed in detail.

This study has a few limitations. First, the sample size was relatively small. Besides, though ADHD can be divided into inattention, hyperactive and combined ADHD subtype, the difference between subtypes was not analyzed in the present study. Therefore, extensive studies with larger populations and long-term follow-up analysis are needed to further confirm the effectiveness of T1, T2 mapping in understanding ADHD GM microstructure changes.

\section{Conclusion}

This study characterized the T1, T2 values of whole-brain GM in drug-naïve pediatric ADHD patients. Alterations of $T 1, T 2$ values were found in ADHD children, in particular GM microstructure changes, possibly concerning iron deficiency, low myelin content, and enlarge vascular interstitial space. We believe these findings could further the understanding of the pathophysiology and clinical manifestations of $A D H D$, thus enabling a more precise and quantitative analysis of this condition. Future longitudinal studies with follow-up of ADHD patients are needed to further verify the partial or complete explanation of these brain changes.

\section{Abbreviations}

ADHD: attention-deficit/hyperactivity disorder

HC: healthy control

GM: grey matter

SyMRI: Synthetic magnetic resonance imaging

DMN: default mode network

EAN: attention and execution control network

\section{Declarations}

\section{Acknowledgments}

We would like to thank the participants and their families, as well as the staff at the MRI at the First Affiliated Hospital of Sun Yat-sen University, for making this study possible. 


\section{Ethics approval}

This study was approved by the institutional review board of the First Affiliated Hospital of Sun Yat-sen University (No. [2019]328).

\section{Consent to participate}

Written informed consent was obtained from the guardians of all the subjects (patients) in this study.

\section{Consent to Publish}

All authors are approval of final version to be published.

\section{Authors Contributions}

Author contributions included: Conceptualization and study design, Shu Su, Yingqian Chen; Methodology, Shu Su, Yan Dai, Long Qian; Investigation, Shu Su, Liping Lin, Qin Zhou, Mengsha Zou, Hongyu Zhang, Meina Liu, Xianhong Xiang; Formal Analysis, Shu Su, Liping Lin; Writing - Original Draft, Shu Su, Yingqian Chen; Writing - Review \& Editing, Shu Su, Yingqian Chen, Zhiyun Yang, Long Qian, Qin Zhou, Mengsha Zou, Hongyu Zhang, Meina Liu, Xianhong Xiang; Visualization, Shu Su. and agreement to be accountable for the integrity and accuracy of all aspects of the work (All authors).

\section{Funding}

This work was supported by the Natural Science Fund Youth Science Fund Project of China [grant numbers 82001439] and the Medical Scientific Research Foundation of Guangdong Province [grant numbers A2020327].

\section{Conflict of interest}

None. The authors of this manuscript declare no relationships with any companies whose products or services may be related to the article's subject matter.

\section{References}

1. Thapar, A., \& Cooper M.Attention deficit hyperactivity disorder[J].The Lancet,2016. 387(10024): $1240-1250$.

2. Thomas, R., Sanders, S., Doust, J., et al.Prevalence of attention-deficit/hyperactivity disorder: a systematic review and meta-analysis[J].Pediatrics,2015. 135(4): e994-1001.

3. Caye, A., Rocha, T. B., Anselmi, L., et al.Attention-Deficit/Hyperactivity Disorder Trajectories From Childhood to Young Adulthood: Evidence From a Birth Cohort Supporting a Late-Onset Syndrome[J].JAMA Psychiatry,2016. 73(7): 705-12. 
4. Ambrosino, S., de Zeeuw, P., Wierenga, L. M., et al.What can Cortical Development in AttentionDeficit/Hyperactivity Disorder Teach us About the Early Developmental Mechanisms Involved? [J].Cereb Cortex,2017. 27(9): 4624-4634.

5. Hoogman, M., Bralten, J., Hibar, D. P., et al.Subcortical brain volume differences in participants with attention deficit hyperactivity disorder in children and adults: a cross-sectional megaanalysis[J].Lancet Psychiatry,2017. 4(4): 310-319.

6. Samea, F., Soluki, S., Nejati, V., et al.Brain alterations in children/adolescents with ADHD revisited: A neuroimaging meta-analysis of 96 structural and functional studies[J].Neurosci Biobehav Rev,2019. 100: $1-8$.

7. Jiang, W. H., Duan, K. K., Chen, J. Y., et al.Structural Brain Alterations and Their Association with Cognitive Function and Symptoms in Attention-Deficit/Hyperactivity Disorder Families[J].European Neuropsychopharmacology,2019. 29: 1189-1190.

8. Cao, M., Shu, N., Cao, Q., et al.Imaging functional and structural brain connectomics in attentiondeficit/hyperactivity disorder[J].Mol Neurobiol,2014. 50(3): 1111-23.

9. Edwards, L. J., Kirilina, E., Mohammadi, S., et al.Microstructural imaging of human neocortex in vivo[J].Neuroimage,2018. 182: 184-206.

10. Lorio, S., Kherif, F., Ruef, A., et al.Neurobiological origin of spurious brain morphological changes: A quantitative MRI study[J].Hum Brain Mapp,2016. 37(5): 1801-15.

11. Deoni, S. C., Zinkstok, J. R., Daly, E., et al.White-matter relaxation time and myelin water fraction differences in young adults with autism[J].Psychol Med,2015. 45(4): 795-805.

12. Megna, R., Alfano, B., Lanzillo, R., et al.Brain tissue volumes and relaxation rates in multiple sclerosis: implications for cognitive impairment[J].J Neurol,2019. 266(2): 361-368.

13. Kupeli, A., Kocak, M., Goktepeli, M., et al.Role of T1 mapping to evaluate brain aging in a healthy population[J].Clin Imaging,2020. 59(1): 56-60.

14. Stuber, C., Morawski, M., Schafer, A., et al.Myelin and iron concentration in the human brain: a quantitative study of MRI contrast[J].Neuroimage,2014. 93 Pt 1: 95-106.

15. Does, M. D.Inferring brain tissue composition and microstructure via MR relaxometry[J].Neuroimage,2018. 182: 136-148.

16. Warntjes, J. B., Leinhard, O. D., West, J., et al.Rapid magnetic resonance quantification on the brain: Optimization for clinical usage[J].Magn Reson Med,2008. 60(2): 320-9.

17. Andica, C., Hagiwara, A., Hori, M., et al.Review of synthetic MRI in pediatric brains: Basic principle of MR quantification, its features, clinical applications, and limitations[J].J Neuroradiol,2019. 46(4): 268-275.

18. Hagiwara, A., Hori, M., Yokoyama, K., et al.Utility of a Multiparametric Quantitative MRI Model That Assesses Myelin and Edema for Evaluating Plaques, Periplaque White Matter, and Normal-Appearing White Matter in Patients with Multiple Sclerosis: A Feasibility Study[J].American Journal of Neuroradiology,2017. 38(2): 237-242. 
19. Norman, L. J., Carlisi, C., Lukito, S., et al.Structural and Functional Brain Abnormalities in AttentionDeficit/Hyperactivity Disorder and Obsessive-Compulsive Disorder: A Comparative Metaanalysis[J].JAMA Psychiatry,2016. 73(8): 815-825.

20. Frodl, T., \& Skokauskas N.Meta-analysis of structural MRI studies in children and adults with attention deficit hyperactivity disorder indicates treatment effects[J].Acta Psychiatr Scand,2012. 125(2): 114-26.

21. Hoogman, M., Bralten, J., Hibar, D. P., et al.Subcortical brain volume differences in participants with attention deficit hyperactivity disorder in children and adults: a cross-sectional megaanalysis[J].Lancet Psychiatry,2017. 4(4): 310-319.

22. Bralten, J., Greven, C. U., Franke, B., et al.Voxel-based morphometry analysis reveals frontal brain differences in participants with ADHD and their unaffected siblings[J].Journal of Psychiatry \& Neuroscience,2016. 41(4): 272-279.

23. Wardlaw, J. M., Benveniste, H., Nedergaard, M., et al.Perivascular spaces in the brain: anatomy, physiology and pathology[J].Nat Rev Neurol,2020. 16(3): 137-153.

24. Wang, Y., Sun, K., Liu, Z., et al.Classification of Unmedicated Bipolar Disorder Using Whole-Brain Functional Activity and Connectivity: A Radiomics Analysis[J].Cereb Cortex,2020. 30(3): 1117-1128.

25. Lei, X., Wang, Y., Yuan, H., et al.Neuronal oscillations and functional interactions between resting state networks[J].Hum Brain Mapp,2014. 35(7): 3517-28.

26. Posner, J., Park, C., \& Wang, Z.Connecting the dots: a review of resting connectivity MRI studies in attention-deficit/hyperactivity disorder[J].Neuropsychol Rev,2014. 24(1): 3-15.

27. Sun, L., Cao, Q., Long, X., et al.Abnormal functional connectivity between the anterior cingulate and the default mode network in drug-naïve boys with attention deficit hyperactivity disorder[J].Psychiatry Res,2012. 201(2): 120-7.

28. Albajara Saenz, A., Villemonteix, T., \& Massat, I.Structural and functional neuroimaging in attentiondeficit/hyperactivity disorder[J].Dev Med Child Neurol,2019. 61(4): 399-405.

29. Vogt, B. A.Cingulate impairments in ADHD: Comorbidities, connections, and treatment[J].Handb Clin Neurol,2019. 166: 297-314.

\section{Figures}



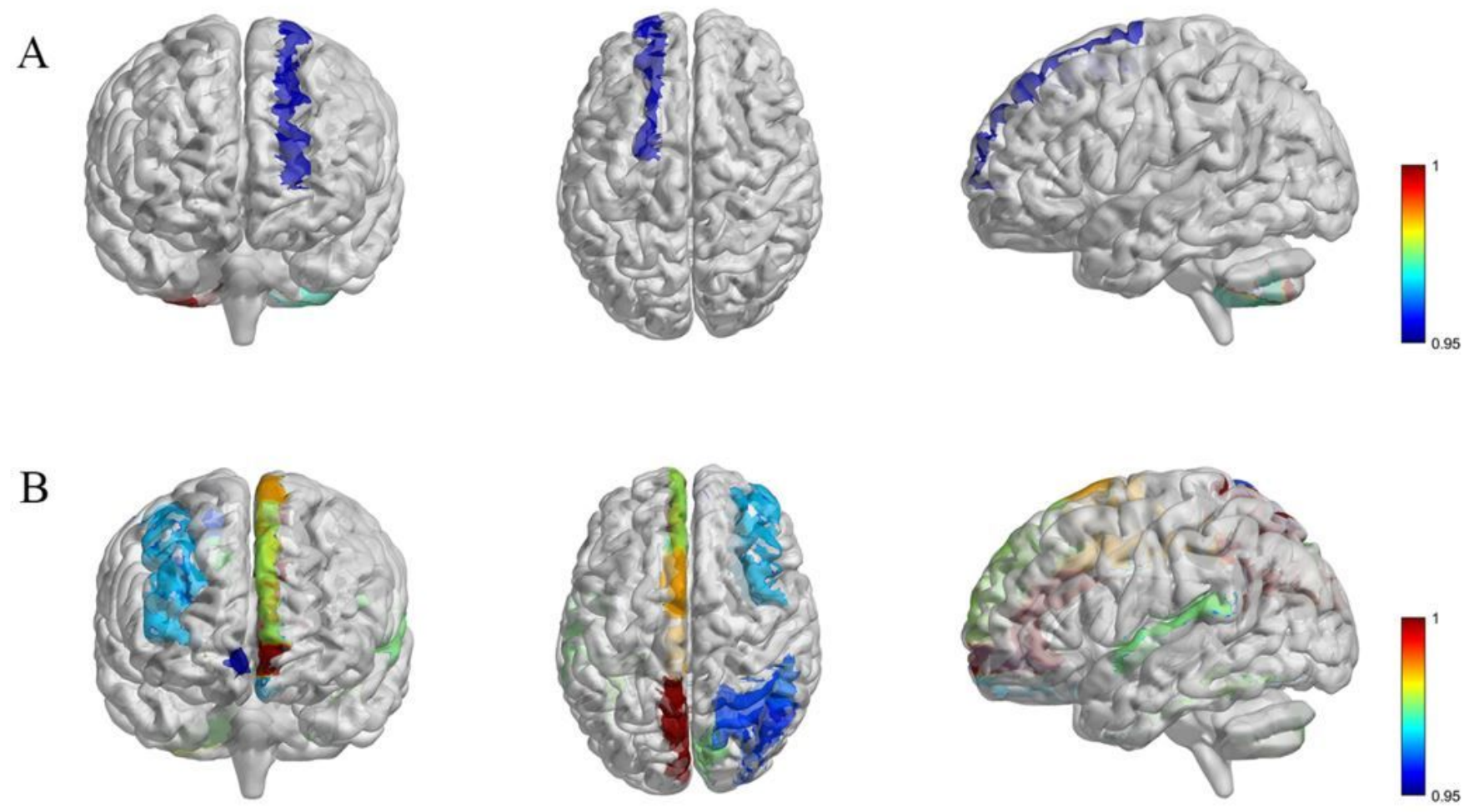

Figure 1

The brain regions with group difference in T1 $(A)$ and T2 $(B)$ values between ADHD and healthy controls $(P<0.05$, FDR-corrected). The detailed information could be found in Table 3. 
A
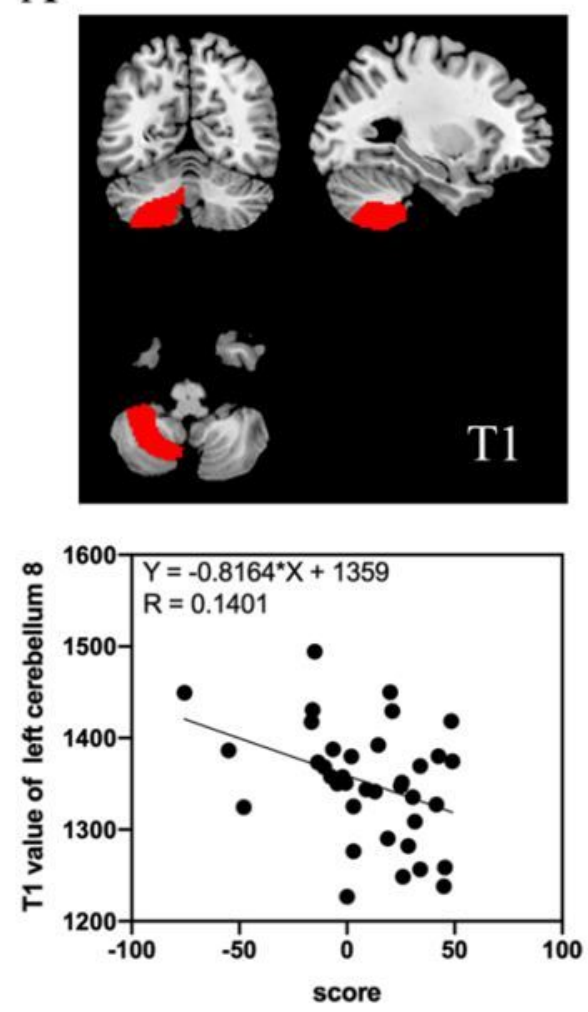

B
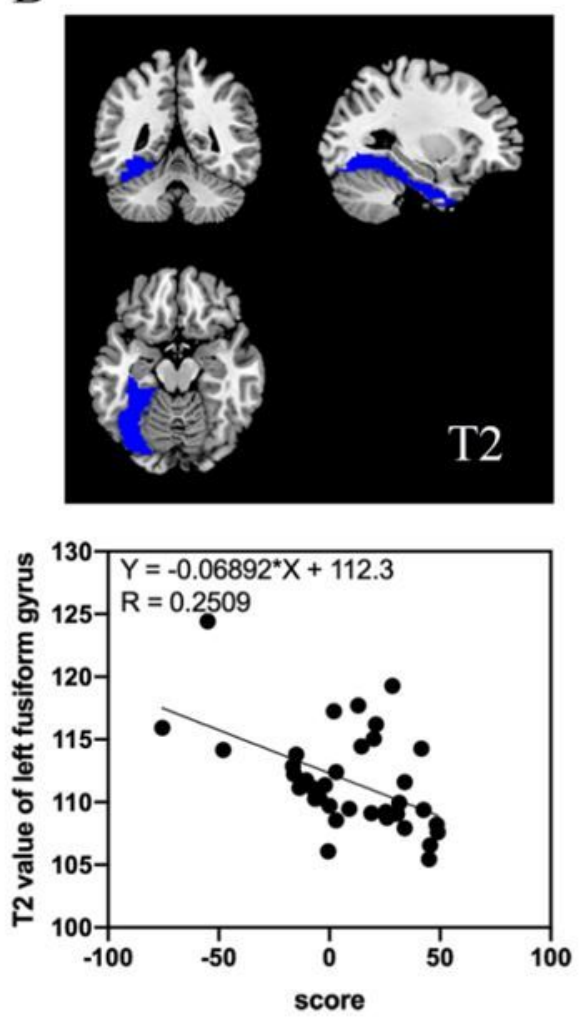

$\mathrm{C}$
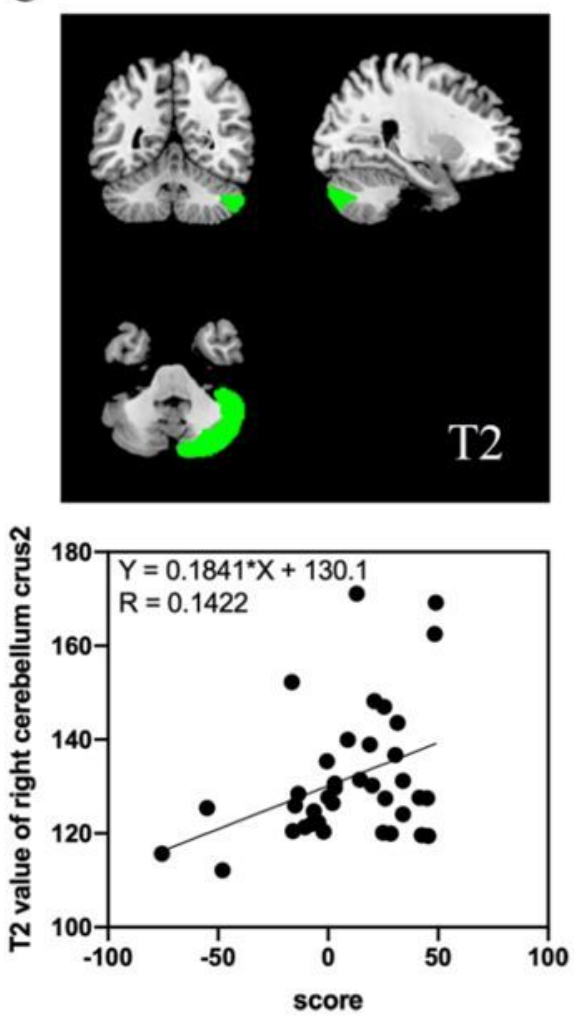

Figure 2

Relationship between the significant group difference regions and clinical variable in ADHD group. The results of correlation analysis between the cancellation test score (x-axis) and T1 map in the left cerebellum $8(R=0.1401 ; P=0.0225)(A)$, and T2 map in the left fusiform gyrus $(R=0.2509 ; P=0.0016)$ $(B)$, as well as T2 map in right cerebellum crus2 $(R=0.1422 ; P=0.0214)(C)$. The corresponding regions were illustrated in the above. 